\title{
Especies nativas del matorral espinoso del noreste mexicano con posibilidades de aprovechamiento industrial
}

\author{
María de los Ángeles Rechy Carvajal ${ }^{1}$ \\ Emil von Roth Rechy²
}

\begin{abstract}
RESUMEN
Las especies maderables del matorral mexicano han sido menospreciadas para su uso comercial, utilizándose mayoritariamente para cercas, leña y carbón, y en menor grado para la fabricación de muebles rústicos. Con este trabajo se pretende ampliar el reducido campo de aprovechamiento industrial de la madera de cuatro especies típicas del matorral espinoso del noreste de México a través del estudio de su anatomía microscópica y la determinación de sus propiedades físico-mecánicas. Las especies estudiadas son barreta (Helietta parvifolia (Gray ex Hemsl.) Benth), canelón (Melia azedarach L.), ébano (Ebenopsis ebano (Berl.) Barneby \& Grimes) y tenaza (Havardia pallens (Benth.) Britt. \& Rose). Las propiedades físico-mecánicas determinadas fueron densidad, porcentaje de contracción y expansión, módulo de elasticidad y resistencia a la flexión (módulo de ruptura, MOR). Los resultados muestran que la barreta es una madera de densidad alta, flexible y de fácil secado caracterizado por una contracción baja. El canelón exhibe una densidad comparativa mayor a la que se observa comúnmente en el pino (a pesar de su crecimiento rápido) teniendo anillos anuales anchos, bajo porcentaje de contracción, alta resistencia al ataque de insectos y siendo su secado fácil, tal como lo es su trabajabilidad. El ébano muestra tener una alta densidad, volviéndose muy duro durante su secado (a tal grado que solo tiene buena trabajabilidad cuando se encuentra en condición verde), agrietándose si el secado se realiza de manera rápida. El duramen de esta especie es muy resistente al ataque de insectos. La tenaza es de fácil secado y tiene alta flexibilidad en condición verde, siendo de baja resistencia al ataque de insectos.
\end{abstract}

PALABRAS CLAVE:

Anatomía microscópica, contracción, densidad, expansión, matorral, módulo de elasticidad.

\begin{abstract}
Brushwood species have been spurned traditionally, their use being limited to the production of fences, firewood and charcoal. This paper presents research results on the microscopical anatomy and physical-mechanical properties of four brushwood species typically found in Northeastern Mexico, with the purpose of expanding the possibility of their commercial use. The species are barreta (Helietta parvifolia (Gray ex Hemsl.) Benth), canelon (Melia azedarach L.), ebony (Ebenopsis ebano (Berl.) Barneby \& Grimes), and tenaza (Havardia pallens (Benth.) Britt. \& Rose). The physical-mechanical properties determined are density, percentage of shrinkage and swelling, modulus of elasticity, and flexural strength. Test results for baretta prove that its wood is heavy, hard, easy to dry, and flexible with little shrinkage during the drying process. Canelon (also known as chinaberry or umbrella tree) is characterized by having a higher density compared to other species. This wood has wide annual growth rings, being easy to dry and work with, and resistant to insect attack. Ebony exhibits a heavy wood that it is only possible to work while green or through special machinery when dry. Ebony's heartwood has a low susceptibility to insect infestations. Shrinkage is similar to that of pine wood (sometimes slightly lower), it is easy to dry, very flexible when wet, however not very resistant to insect infestations.
\end{abstract}

KERWORDS:

Microscopical anatomy, shrinkage, density, swelling, brushwood, modulus of elasticity. 


\section{INTRODUCCIÓN}

La zona semidesértica de México abarca una gran porción de la superficie de la República Mexicana, comprendiendo un $29,3 \%$ de ésta. Esta área es igual a la suma de áreas de bosques de clima templado y de selvas, comprendiendo $15,5 \%$ y $13,4 \%$ respectivamente. La vegetación predominante en esta zona corresponde a la del matorral espinoso, cuyas especies maderables son sujetas a un pobre aprovechamiento industrial. Las especies arbóreas de este trabajo se caracterizan típicamente por un crecimiento lento debido a la escasez de agua y por exhibir una densidad comparativamente mayor a la del pino. En el caso del ébano, una de las maderas más abundantes del matorral (Villalón, 1989), se ha utilizado primordialmente para la producción de carbón. Este uso no representa el aprovechamiento más adecuado, dado que aún siguiendo procesos de producción optimizados, solamente se puede obtener entre $20 \mathrm{~kg}$ a $25 \mathrm{~kg}$ de carbón de cada $100 \mathrm{~kg}$ de madera (Comunicación directa, Aserradero El 18, Linares, Nuevo León). El ébano también se utiliza, aunque en menor escala, para la fabricación de muebles. Desafortunadamente, la industria actual no utiliza procesos de producción adecuados, mermándose la calidad de los muebles que de otra manera se podrían exportar. En el caso del ébano, se puede observar por lo general un proceso de secado incorrecto que se traduce en la aparición de grietas no deseadas en madera decorativa. Otras de las maderas más abundantes de esta región son la barreta, la tenaza y el canelón (Villalón, 1989). Estas maderas comparten con el ébano, a pesar de sus diferentes características, el mismo tipo de aprovechamiento industrial debido a la falta de investigación de sus características individuales y su divulgación respectiva.
Existe poca información acerca de estudios realizados sobre las especies maderables de la región noreste de México. Entre aquellas con relación directa a esta investigación destacan los trabajos de Wolf y Perales (1985), que investigaron la resistencia de varías especies al ataque de microorganismos; Jurado y Reid (1989), que realizaron un estudio de las especies más abundantes y su localización geográfica; Estrada y Marroquín (1990), que desarrollan un estudio sobre identificación y localización de numerosas especies; Infante Mercado (1993), quien investigó las propiedades físico-mecánicas de una especie de mezquite; Hernández Guevara (1997), en el que se realiza un análisis estructural de la barreta; Rechy y von Roth (1996), que aborda las propiedades físico-mecánicas del ébano para su aprovechamiento industrial; Rechy (1998), quien realizó un estudio tecnológico de varias especies del matorral con el objetivo de aprovechar las maderas para la manufactura de instrumentos musicales de cuerdas talladas; y el de Rechy y Domínguez (2001), que trabajaron en la tecnología de manufactura de violines de alta calidad empleando maderas mexicanas (incluyéndose algunas del matorral espinoso).

\section{OBJETIVOS}

La presente investigación analiza cuatro especies maderables del matorral espinoso de la región semidesértica del noreste de México determinando su anatomía microscópica y diversas propiedades físico-mecánicas con el objetivo de optimizar su aprovechamiento industrial. Las especies sujetas a la investigación fueron barreta (Helietta parvifolia (Gray ex Hemsl.) Benth), canelón (Melia azedarach L.), ébano (Ebenopsis ebano (Berl.) Barneby \& Grimes) y tenaza (Havardia pallens (Benth.) Britt. \& Rose). 


\section{METODOLOGÍA}

Las maderas estudiadas se colectaron en los predios de la Facultad de Ciencias Forestales de la Universidad Autónoma de Nuevo León, campus Linares, así como en sus alrededores. El municipio de Linares está ubicado en el centro sur del estado de Nuevo León, limitado por las coordenadas $25,13^{\circ}$ y $24,32^{\circ}$ de latitud norte y $100,0^{\circ}-99,07^{\circ}$ de longitud oeste. La altura sobre el nivel del mar es de 350 metros. Los suelos son predominantemente vertisoles y regosoles. La precipitación anual promedio es de $750 \mathrm{~mm}$ con una desviación estándar de $250 \mathrm{~mm}$ y una media de 80 precipitaciones anuales, de las cuales el $60 \%$ es de menos de $5 \mathrm{~mm}$. La temperatura promedio es de $21^{\circ} \mathrm{C}$ (Navar Cháidez, 2003).

Se colectaron cuatro individuos de cada una de las especies en el área anteriormente descrita. Se cortó un segmento longitudinal de $100 \mathrm{~cm}$ de tronco de cada árbol con un diámetro de entre $5 \mathrm{~cm}$ y 10 $\mathrm{cm}$ en dicho segmento, esto a partir de una altura de $50 \mathrm{~cm}$ sobre el nivel del suelo para evitar variaciones de composición de madera que son inherentes a la cercanía a éste. En las secciones transversales extremas se aplicó, en campo, una capa de pintura impermeable con el fin de minimizar la pérdida de humedad no uniforme que se concentra típicamente en las secciones de corte. Las muestras se dejaron secar a una temperatura que osciló entre $20{ }^{\circ} \mathrm{C}$ y $25^{\circ} \mathrm{C}$ y a una humedad relativa del aire de $65 \%$ hasta que la madera alcanzó un contenido de humedad de entre $12 \%$ y $13 \%$.

Se realizó la identificación botánica por especialistas de la Facultad de Ciencias Forestales en Linares, incorporándose las ejemplares correspondientes al herbario de la misma. Posteriormente se cortaron, para el estudio de anatomía microscópica, 10 rodajas de $2 \mathrm{~cm}$ de espesor de cada uno de los 16 segmentos (4 individuos por especie), siendo cortadas a lo largo toda la longitud de los segmentos, sin concentrar los cortes a una altura particular. De estas rodajas se cortaron cubos de $2 \mathrm{~cm}$ de arista en las direcciones correspondientes al estudio anatómico microscópico (transversal, radial y tangencial). Para el ablandamiento, se siguió la práctica de cocción de las muestras en agua hasta obtener una consistencia tal que fuera posible la fácil obtención de laminillas a través de un microtomo marca Leica RM 2125. La evaluación cualitativa de sustancias extraíbles se determinó durante el ablandamiento anteriormente descrito de las muestras. La coloración del agua durante la cocción se relacionó directamente con el volulmen de sustancias extraíbles (a mayor color, mayor cantidad de éstas). Se utilizaron como colorantes Safranina y azul de Astra al 1 $\%$ en solución alcohólica al 60 \% para aumentar los contrastes en las laminillas. La observación y obtención de imágenes microscópicas se realizó mediante un fotomicroscopio Zeiss II, con un ocular 10x.

Para la obtención de propiedades físico-mecánicas se siguieron tres diferentes tipos de normas con el propósito de comparación de resultados: ASTM, ISO y DIN. El tamaño de las probetas varió dependiendo del ensayo realizado y la norma aplicada. En todas las pruebas se utilizaron 20 probetas por especie, proviniendo éstas de los troncos cortados en campo (5 probetas de cada fuste). Para la determinación de contenido de humedad se utilizaron probetas con dimensiones de $20 \mathrm{~mm} \times 20 \mathrm{~mm} \times 10 \mathrm{~mm}$ (DIN 52183 y ASTM D143) (DIN, 1992c; ASTM, 2003), así como probetas con dimensiones de $20 \times 20 \times 25 \mathrm{~mm}$ (ISO 3130) (DIN, 1992d). Para la determinación de la densidad se utilizaron probetas de $30 \mathrm{~mm} \times 30 \mathrm{~mm} \times 100 \mathrm{~mm}$ (DIN 
52182) (DIN, 1992b), $20 \mathrm{~mm} \times 20 \mathrm{~mm} \times$ $25 \mathrm{~mm}$ (ISO 3131) (DIN, 1992E) y de 50 $\mathrm{mm} \times 50 \mathrm{~mm} \times 150 \mathrm{~mm}$ (ASTM D143) (ASTM, 2003). Las probetas de $30 \mathrm{~mm} x$ $30 \mathrm{~mm} \times 100 \mathrm{~mm}$ que se utilizaron para los ensayos de densidad DIN se utilizaron para determinar la expansión y contracción volumétrica. Las pruebas de módulo de elasticidad y resistencia a flexión se llevaron a cabo siguiendo las normas DIN 52180, ISO 3133 у ASTM 143 (DIN, 1992a; 1992f; ASTM, 2003).

Los diferentes ensayos llevados a cabo en esta investigación demostraron diversas caracTerísticas cualitativas de las especies estudiadas (rapidez de secado a través de las pruebas de determinación de contenido de humedad, así como concentración y expansión volumétrica; trabajabilidad al cortar las probetas de los troncos, etc.). Estas características se exponen en las conclusiones de esta publicación. Cabe destacar que el ataque de la madera por insectos se determinó exponiendo la madera a la intemperie durante un periodo de tres meses en los predios de la Facultad de Ciencias Forestales, UANL. El ataque se determinó por inspección visual de la madera degradada, pudiéndose relacionar la condición de ésta con las características típicas de ataque de los diferentes tipos de insectos. Pruebas reglamentadas por normas tecnológicas que sustenten las observaciones sobre el ataque de insectos están fuera del alcance de esta investigación, por lo que se incluyen éstas como características (no cuantificadas) de las maderas.

\section{RESULTADOS}

Las tablas 1, 2 y 3 muestran los resultados estadísticos del análisis de anatomía microscópica, detallando diferentes características de las fibras, vasos y rayos de la estructura de cada especie. Las tablas 4,5 y 6 muestran características adicionales de anatomía microscópica. Las tablas 7 a 11 resumen los resultados de las pruebas físico-mecánicas. Los resultados presentados de ensayos estandarizados corresponden a los de las normas DIN.

La comparación de resultados de las tres diferentes normas mostró que estos eran prácticamente iguales en sus valores de contenido de humedad y densidad. Al igual que en los ensayos de

Tabla 1. Espesor de pared de fibras (micras)

\begin{tabular}{lccccc}
\hline VALOR & BARRETA & ÉBANO & \multicolumn{2}{c}{ TENAZA } & CANELÓN \\
\cline { 3 - 5 } & & & $\begin{array}{c}\text { Madera } \\
\text { temprana }\end{array}$ & $\begin{array}{c}\text { Madera } \\
\text { tardía }\end{array}$ & \\
\hline Promedio & 4,41 & 4,58 & 1,764 & 3,027 & 3,257 \\
\hline Máximo & 4,749 & 5,437 & 2,714 & 4,071 & 5,428 \\
\hline Mínimo & 4,071 & 3,392 & 1,357 & 1,357 & 1,357 \\
\hline $\begin{array}{l}\text { Desviación } \\
\text { estándar }\end{array}$ & 0,357 & 0,691 & 0,655 & 0,764 & 1,144 \\
\hline
\end{tabular}


Tabla 2. Diámetro de vasos (micras)

\begin{tabular}{lcccc}
\hline VALOR & BARRETA & ÉBANO & TENAZA & CANELÓN \\
\hline Promedio & 33,51 & 125,3 & 116,7 & 85,5 \\
\hline Máximo & 48,75 & 155,25 & 159,9 & 132,5 \\
\hline Mínimo & 27,3 & 101 & 53,33 & 42,1 \\
\hline Desviación estándar & 3,655 & 18,57 & 27,71 & 26,6 \\
\hline
\end{tabular}

Tabla 3. Longitud (L) y espesor (e) de radios (micras)

\begin{tabular}{lllcccc}
\hline \multirow{2}{*}{ Barreta } & Uniseriados & $\mathrm{L}$ & 54,38 & 70,2 & 31,2 & 14,26 \\
\cline { 3 - 7 } & & $\mathrm{t}$ & 10,13 & 13,64 & 7,8 & 1,97 \\
\cline { 3 - 7 } & Biseriados & $\mathrm{L}$ & 126,9 & 148,2 & 103,3 & 18,5 \\
\cline { 3 - 7 } & $\mathrm{t}$ & 18,33 & 21,45 & 13,65 & 2,55 \\
\hline \multirow{2}{*}{ Ébano } & Uniseriados & $\mathrm{L}$ & 163,8 & 169,9 & 107,2 & 152,7 \\
\cline { 3 - 7 } & & $\mathrm{t}$ & 6,75 & 8,3 & 6,1 & 0,758 \\
\hline \multirow{2}{*}{ Tenaza } & Uniseriados & $\mathrm{L}$ & 141,99 & 199,99 & 70,99 & 39,13 \\
\cline { 3 - 7 } & & $\mathrm{t}$ & 15,33 & 19,99 & 13,33 & 3,22 \\
\hline \multirow{2}{*}{ Canelón } & Uniseriados & $\mathrm{L}$ & 262,7 & 378,4 & 175,5 & 5,102 \\
\cline { 3 - 7 } & & $\mathrm{t}$ & 37,63 & 58,5 & 25,35 & 103,1 \\
\hline
\end{tabular}

Tabla 4. Diámetro de fibras (micras)

\begin{tabular}{lccccc}
\hline PARÁMETRO & BARRETA & ÉBANO & \multicolumn{2}{c}{ TENAZA } & CANELÓN \\
\cline { 3 - 5 } & & & $\begin{array}{c}\text { Madera } \\
\text { temprana }\end{array}$ & $\begin{array}{c}\text { Madera } \\
\text { tardía }\end{array}$ & \\
\hline Diámetro & 12,213 & 11,671 & 16,013 & 1,483 & 19,27 \\
\hline $\begin{array}{l}\text { Diámetro de } \\
\text { lumen }\end{array}$ & 3,392 & 2,51 & 12,485 & 5,428 & 12,756 \\
\hline
\end{tabular}


Tabla 5. Densidad de población de vasos

\begin{tabular}{|c|c|c|c|c|c|}
\hline \multirow[t]{2}{*}{ VALOR } & \multirow[t]{2}{*}{ BARRETA } & \multirow[t]{2}{*}{ ÉBANO } & \multicolumn{2}{|c|}{ TENAZA } & \multirow[t]{2}{*}{ CANELÓN } \\
\hline & & & $\begin{array}{c}\text { Madera } \\
\text { temprana }\end{array}$ & $\begin{array}{c}\text { Madera } \\
\text { tardía }\end{array}$ & \\
\hline $\begin{array}{l}\text { Densidad } \\
\text { (número/mm²) }\end{array}$ & 80,1 & $20-22$ & $18-20$ & $20-26$ & $10-12$ \\
\hline
\end{tabular}

Tabla 6. Características de radios según especie

\begin{tabular}{lcccc}
\hline PARÁMETRO & BARRETA & ÉBANO & TENAZA & CANELÓN \\
\cline { 2 - 5 } & $\begin{array}{c}\text { Uni- } y \\
\text { Biseriados }\end{array}$ & Uniseriados & Uniseriados & $\begin{array}{c}\text { Bi- } y \\
\text { Triseriados }\end{array}$ \\
\hline $\begin{array}{l}\text { Densidad } \\
\text { (número/mm }\end{array}$ & $20-25$ & $50-55$ & $20-26$ & $14-16$ \\
\hline Distribución & Difusa & Difusa & Difusa & Difusa \\
\hline $\begin{array}{l}\text { Sustancias } \\
\text { extraíbles }\end{array}$ & Pocas & $\mathrm{Si}$ & Muy pocas & $\mathrm{Si}$ \\
\hline Cristales & $\mathrm{Si}$ & $\mathrm{No}$ & $\mathrm{Si}$ & $\mathrm{No}$ \\
\hline
\end{tabular}

Tabla 7. Densidad de madera según especie $\left(\mathrm{g} / \mathrm{cm}^{3}\right)$

\begin{tabular}{lcccc}
\hline VALOR & BARRETA & ÉBANO & TENAZA & CANELÓN \\
\hline Promedio & 0,91 & 0,98 & 0,5 & 0,68 \\
\hline Máximo & 0,99 & 1,1 & 0,57 & 0,73 \\
\hline Mínimo & 0,91 & 0,89 & 0,43 & 0,66 \\
\hline $\begin{array}{l}\text { Desviación } \\
\text { estándar }\end{array}$ & 0,0337 & 0,0791 & 0,0647 & 0,0254 \\
\hline
\end{tabular}


Tabla 8. Contracción volumétrica (\%)

\begin{tabular}{lcccc}
\hline VALOR & BARRETA & ÉBANO & TENAZA & CANELÓN \\
\hline Promedio & 2,03 & 5,64 & 2,94 & 5,49 \\
\hline Máximo & 2,81 & 6,33 & 4,1 & 6,42 \\
\hline Mínimo & 1,04 & 4,89 & 1,5 & 2,83 \\
\hline $\begin{array}{l}\text { Desviación } \\
\text { estándar }\end{array}$ & 0,90 & 0,49 & 0,84 & 1,02 \\
\hline
\end{tabular}

Tabla 9. Expansión volumétrica según especie (\%)

\begin{tabular}{lcccc}
\hline VALOR & BARRETA & ÉBANO & TENAZA & CANELÓN \\
\hline Promedio & 4,50 & 12,21 & 12,81 & 9,85 \\
\hline Máximo & 7,04 & 14,75 & 15,8 & 10,8 \\
\hline Mínimo & 2,77 & 10,69 & 11,4 & 7,9 \\
\hline $\begin{array}{l}\text { Desviación } \\
\text { estándar }\end{array}$ & 1,75 & 1,27 & 1,77 & 0,80 \\
\hline
\end{tabular}

Tabla 10. Módulo de elasticidad $\left(\mathrm{kN} / \mathrm{mm}^{2}\right)$

\begin{tabular}{lcccc}
\hline VALOR & BARRETA & ÉBANO & TENAZA & CANELÓN \\
\hline Promedio & 10258 & 10397 & 6804 & 8591 \\
\hline Máximo & 10431 & 10470 & 7263 & 9522 \\
\hline Mínimo & 9997 & 10291 & 6575 & 7548 \\
\hline $\begin{array}{l}\text { Desviación } \\
\text { estándar }\end{array}$ & 245,95 & 94,12 & 485,78 & 678,52 \\
\hline
\end{tabular}

Tabla 11. Resistencia a flexión (Módulo de ruptura, $\mathrm{kN} / \mathrm{mm}^{2}$ )

\begin{tabular}{lcccc}
\hline VALOR & BARRETA & ÉBANO & TENAZA & CANELÓN \\
\hline Promedio & 144,02 & 95,71 & 82,04 & 97,91 \\
\hline Máximo & 168,32 & 103,471 & 82,21 & 119,84 \\
\hline Mínimo & 112,07 & 83,96 & 81,88 & 73,92 \\
\hline $\begin{array}{l}\text { Desviación } \\
\text { estándar }\end{array}$ & 28,896 & 10,352 & 0,233 & 17,28 \\
\hline
\end{tabular}


contenido de humedad y densidad, se observaron de nueva cuenta valores prácticamente iguales.

\section{DISCUSIÓN Y CONCLUSIONES}

Los diferentes ensayos llevados a cabo en esta investigación demostraron los siguientes rangos cualitativos de las maderas estudiadas. La barreta es dura, de pulido difícil, cepillado fácil y de la que se pueden obtener con mucha facilidad chapas en corte tangencial. Tiene un veteado visualmente atractivo, es fácil de secar y es resistente al ataque de insectos. El ébano, al igual que la barreta, tiene una madera dura, de pulido difícil y cepillado fácil. Sus chapas se pueden obtener con facilidad hasta espesores de alrededor de $2 \mathrm{~mm}$. El ébano presenta un secado rápido, sin embargo se caracteriza por numerosas grietas. El duramen es resistente al ataque de insectos, no así la albura. La tenaza es dura y de lijado difícil que produce un polvo que ataca la mucosa bucal y nasal, produciendo estornudos e irritación al entrar en contacto con la piel. Es de un cepillado fácil y las chapas se pueden obtener en espesores muy delgados. Su madera presenta poca resistencia al ataque de insectos. El canelón tiene una madera dura, con buena trabajabilidad (lijado, cepillado y cortado) y de la cual se pueden obtener fácilmente chapas de espesores normales. Su secado es fácil, dado que no presenta grietas durante éste. Es resistente al ataque de insectos. La madera del canelón se parece a la del cedro mexicano. La observación de especímenes de canelón plantados en la Facultad de Ciencias Forestales (UANL) sugiere que éste es de crecimiento rápido cuando cuenta con riego de manera regular, lográndose diámetros de $25 \mathrm{~cm}$ a $30 \mathrm{~cm}$ después de 8 años.

Los análisis de anatomía microscópica demostraron que los diámetros de lumen de la tenaza y del canelón son aproximadamente cuatro veces mayor que aquellos observados en la barreta y el ébano. Esto se puede relacionar con el crecimiento rápido de las dos primeras especies y el lento en las otras dos. Los vasos de la barreta son particularmente pequeños, siendo su densidad la más alta de las especies estudiadas. La longitud y el espesor de los rayos del canelón son mayores que en las demás maderas, teniendo por ende la menor densidad de presencia de radios. La longitud y densidad de radios observada en el ébano se traducen en la dificultad que caracteriza el secado de su madera. El bajo contenido de sustancias extraíbles en agua de la tenaza se refleja en la baja resistencia al ataque de insectos, siendo ya esto observado en la prueba cualitativa en campo. Los cristales presentes en la anatomía microscópica de la barreta y la tenaza no demostraron tener influencia en el aserrío y la trabajabilidad de estas maderas.

Los ensayos físico-mecánicos demuestran que la tenaza es la madera con la menor densidad, seguida por el canelón, al cual se le considera de densidad media. La barreta y el ébano poseen las densidades más altas debido a su estructura anatómica y el contenido de sustancias accesorias. Los valores de contracción volumétrica de las cuatro especies son relativamente bajos en comparación con la especie pino (Pinus durangensis), que tiene un valor típico de alrededor del 7,7 \%. El porcentaje de expansión volumétrica sigue un patrón parecido al de la contracción, teniendo las especies estudiadas valores de entre $8 \%$ y $10 \%$, mientras la especie de pino anteriormente citada tiene un valor de 14,2\%. La barreta y el ébano presentaron los módulos de elasticidad más altos, confirmando los valores obtenidos para las 4 especies la relación directa entre el valor del módulo de elasticidad y la densidad. 
La barreta mostró tener el valor de resistencia a flexión más alto, seguida por el canelón y posteriormente por el ébano. Nótese que la varianza en los valores obtenidos para el canelón es considerable.

La comparación de los resultados cuantitativos y cualitativos expuestos anteriormente con las propiedades de las maderas utilizadas comúnmente en la industria, particularmente el pino, muestran que las especies estudiadas cuentan con una calidad muy competitiva. Dado el lento crecimiento de las especies estudiadas (exceptuando al canelón que puede ser de crecimiento rápido bajo riego regular), la madera de éstas puede encontrar amplio uso en aquellos productos para los cuales no son necesarios grandes volúmenes de materia prima. El ébano y la barreta se adecuan para la fabricación de herramientas (cachas de éstas), moldes de fundición, instrumentos musicales, duelas de piso, partes visibles de muebles tapizados, etc., por la alta rigidez (rigidez a flexión, cortante y fuerza axial están en función del módulo de elasticidad) y los valores de resistencia a flexión que presentan. El canelón es idóneo para la fabricación de muebles, de recubrimientos de pared en forma de chapas, así como para figuras talladas dado su rápido crecimiento, la poca cantidad de grietas que desarrolla durante el secado y por su buena trabajabilidad. Se sugiere a la tenaza por sus características de anatomía microscópica y físico-mecánicas anteriormente descritas para la fabricación de maderas laminadas, en particular para marcos de pinturas o fotografías.

Las especies estudiadas demuestran que las maderas del matorral del noreste mexicano tienen propiedades que permiten un aprovechamiento industrial mejor que el que se les está dando hoy en día. Cambios en las prácticas actuales de la industria, que estén de acuerdo con las propiedades características de las especies, se traducen en un uso más eficiente de la materia prima. Si estos cambios van acompañados por programas de reforestación, no sólo se asegura el abasto de madera, sino que también se contrarresta el impacto que la deforestación está teniendo en el ecosistema de esta región.

\section{REFERENCIAS}

ASTM (American Standard Test Methods). 2003. D143-94 (2000) Standard Test Methods for Small Clear Specimens of Timber. P: 4345, 114-115, 122-125. ASTM. EUA.

DIN (Deutsches Institut für Normung eV). 1992a. Norm DIN 52180. Beuth Verlag GmbH. Berlin / Köln, Alemania.

DIN (Deutsches Institut für Normung eV). 1992b. Norm DIN 52182. Beuth Verlag $\mathrm{GmbH}$. Berlin/Köln, Alemania.

DIN (Deutsches Institut für Normung eV). 1992c. Norm DIN 52183. Beuth Verlag $\mathrm{GmbH}$. Berlin/Köln, Alemania.

DIN (Deutsches Institut für Normung eV). 1992d. Normen über Holz Verzeichhnis Internationaler Normen ISO sowie deren Zusammenhang mit DIN. Norm ISO 3130. Beuth Verlag GmbH. Berlin / Köln, Alemania.

DIN (Deutsches Institut für Normung eV). 1992e. Normen über Holz Verzeichhnis Internationaler Normen ISO sowie deren Zusammenhang mit DIN. Norm ISO 3131. Beuth Verlag GmbH. Berlin / Köln, Alemania.

DIN (Deutsches Institut für Normung eV). 1992 f. Normen über Holz Verzeichhnis Internationaler Normen 
ISO sowie deren Zusammenhang mit DIN. Norm ISO 3133. Beuth Verlag GmbH. Berlin / Köln, Alemania.

Estrada E. y Marroquín J. 1990. Leguminosas en el Centro - Sur de Nuevo León. Reporte científico, Num. Especial 10 Fac de C. Forestales UANL.

Hernández Guevara, R. 1997. Análisis estructural e importancia económica de Helietta parvifolia en dos zonas del norte y sureste del estado de Nuevo León. Tesis de licenciatura. Facultad de Ciencias Biológicas, UANL. Monterrey, México.

Infante Mercado, O. 1993. Estudio físicomecánico del mezquite del sur de Nuevo León. Tesis de licenciatura. Facultad de Ciencias Forestales, UANL. Linares, México.

Jurado E., Reid N. 1989. Influencia de factores edáfico- topográficos y perturbación sobre el matorral espinoso o tamaulipeco en linares, N.L. Reporte científico No. 10 Facultad de Ciencias Forestales, UNAL. Linares, México.

Navar Chaidez. J. 2003 Información directa de Control Hidrológico de la Región. Fac. de C. Forestales UANL Linares, N.L.
Rechy M., von Roth, W. 1996. Estudio físico-mecánico del ebano del sur de Nuevo León. Memorias del 1 es Congreso de Tecnología de Productos Forestales. Xalapa, México.

Rechy M. 1998. Estudio anatómico microscópico y físico de varias especies del matorral para instrumentos musicales de cuerdas talladas. Memorias del Simposio de Tecnología de productos Forestales. Morelia, México.

Rechy, M. Domínguez, L.A. 2002. Manufactura de un violín hecho con maderas mexicanas. IV Congreso de Tecnología de Productos Forestales. Guadalajara, México.

Villalón M. H. 1989. Ein Beitrag Zur Verwertung von Biomasseproduktion und deren Qualität für die forts-und landwirtschaftliche Nutzung des Matorrals in der Gemeinde Linares, N.L., Mexico. Tesis Doctoral. Göttingen, Alemenia.

Wolf F., Perales F. 1985. Durabilidad natural de la Madera de algunas especies del matorral del noreste de México. Reporte científico No.3 facultad de Silvicultura y manejo de recursos renovables, UANL. Linares, México.» posibilidades de aprovechamiento industrial. Madera y Bosques 10(2):45-54. 